\title{
Quantum Nonlinear Optics with Single Photons Enabled by Strongly Interacting Atoms
}

\section{Citation}

Peyronel, Thibault, Ofer Firstenberg, Qi-Yu Liang, Sebastian Hofferberth, Alexey V. Gorshkov, Thomas Pohl, Mikhail D. Lukin, and Vladan Vuletić. 2012. Quantum Nonlinear Optics with Single Photons Enabled by Strongly Interacting Atoms. Nature 488, no. 7409: 57-60.

\section{Published Version}

doi:10.1038/nature11361

\section{Permanent link}

http://nrs.harvard.edu/urn-3:HUL.InstRepos:12967681

\section{Terms of Use}

This article was downloaded from Harvard University's DASH repository, and is made available under the terms and conditions applicable to Other Posted Material, as set forth at http:// nrs.harvard.edu/urn-3:HUL.InstRepos:dash.current.terms-of-use\#LAA

\section{Share Your Story}

The Harvard community has made this article openly available.

Please share how this access benefits you. Submit a story.

Accessibility 


\section{Quantum Nonlinear Optics with Single Photons Enabled by Strongly Interacting Atoms}

Thibault Peyronel $^{1}$, Ofer Firstenberg ${ }^{2}$, Qi-Yu Liang ${ }^{1}$, Sebastian Hofferberth ${ }^{2}$, Alexey V. Gorshkov ${ }^{3}$, Thomas Pohl ${ }^{4}$, Mikhail D. Lukin² ${ }^{2}$ and Vladan Vuletić ${ }^{1}$

${ }^{1}$ Department of Physics and Research Laboratory of Electronics, Massachusetts Institute of Technology, Cambridge, MA 02139, USA

${ }^{2}$ Department of Physics, Harvard University, Cambridge, MA 02138, USA

${ }^{3}$ Institute for Quantum Information and Matter, California Institute of Technology, Pasadena, CA 91125, USA

${ }^{4}$ Max Planck Institute for the Physics of Complex Systems, Nöthnitzer Str. 38, D-01187 Dresden, Germany

The realization of strong nonlinear interactions between individual light quanta (photons) is a long-standing goal in optical science and engineering ${ }^{1,2}$ that is both of fundamental and technological significance. In conventional optical materials, the nonlinearity at light powers corresponding to single photons is negligibly weak. Here we demonstrate a medium that is nonlinear at the level of individual quanta, exhibiting strong absorption of photon pairs while remaining transparent to single photons. The quantum nonlinearity is obtained by coherently coupling slowly propagating photons $^{3-5}$ to strongly interacting atomic Rydberg states $^{6-12}$ in a cold, dense atomic gas ${ }^{13,14}$. Our approach opens the door for quantum-byquantum control of light fields, including single-photon switching ${ }^{15}$, all-optical deterministic 


\section{quantum logic ${ }^{16}$, and the realization of strongly correlated many-body states of light ${ }^{17}$.}

Recently, remarkable advances have been made towards optical systems that are nonlinear at the level of individual photons. The most promising approaches have used high-finesse optical cavities to enhance the atom-photon interaction probability ${ }^{2,18-21}$. In contrast, our present method is cavity-free and is based on mapping photons onto atomic states with strong interactions in an extended atomic ensemble ${ }^{13,15,22,23}$. The central idea is illustrated in Fig. 1, where a quantum probe field incident onto a cold atomic gas is coupled to high-lying atomic states (Rydberg levels ${ }^{24}$ ) by means of a second, stronger laser field (control field). For a single incident probe photon, the control field induces a spectral transparency window in the otherwise opaque medium via Electromagnetically Induced Transparency $\left(\mathrm{EIT}^{5}\right)$, and the probe photon travels at much reduced speed in the form of a coupled excitation of light and matter (Rydberg polariton). However, in stark contrast to conventional EIT, if two probe photons are incident onto the Rydberg medium, the strong interaction between two Rydberg atoms tunes the transition out of resonance, thereby destroying the transparency and leading to absorption ${ }^{15,22,23,25,26}$. The experimental demonstration of an extraordinary optical material exhibiting strong two-photon attenuation in combination with single-photon transmission is the central result of this work.

The quantum nonlinearity can be viewed as a photon-photon blockade mechanism that prevents the transmission of any multi-photon state. It arises from the Rydberg excitation blockade ${ }^{27}$, which precludes the simultaneous excitation of two Rydberg atoms that are separated by less than a blockade radius $r_{b}$ (see Figure 1). During the optical excitation under EIT conditions, an inci- 
dent single photon is converted into a Rydberg polariton inside the medium. However, due to the Rydberg blockade, a second polariton cannot travel within a blockade radius from the first one, and EIT is destroyed. Accordingly, if the second photon approaches the single Rydberg polariton, it will be significantly attenuated, provided that $r_{b}$ exceeds the resonant attenuation length of the medium in the absence of EIT, $l_{a}=\left(\mathcal{N} \sigma_{a}\right)^{-1}$, where $\mathcal{N}$ is the peak atomic density and $\sigma_{a}$ the absorption cross-section. This simple physical picture implies that, in the regime where the blockade radius exceeds the absorption length $\left(r_{b} \gtrsim l_{a}\right)$, two photons in a tightly focused beam not only cannot pass through each other ${ }^{15}$, but also cannot propagate close to each other inside the medium (see Fig. 1c and detailed theoretical analysis below). Using Rydberg states with principal quantum numbers $46 \leq n \leq 100$, we can realize blockade radii $r_{b}$ between $3 \mu \mathrm{m}$ and $13 \mu \mathrm{m}$, while for our highest atomic densities of $\mathcal{N}=2 \times 10^{12} \mathrm{~cm}^{-3}$, the attenuation length $l_{a}$ is below $2 \mu \mathrm{m}$. The optical medium then acts as a quantum nonlinear absorption filter, converting incident laser light into a non-classical train of single-photon pulses.

EIT nonlinearities at the few-photon level have been previously observed without using strongly interacting atomic states by means of strong transverse confinement of the light ${ }^{28,29}$. The interactions between cold Rydberg atoms have been explored in ensembles ${ }^{6-10}$, and have been used to realize quantum logic gates between two Rydberg atoms ${ }^{11,12,24}$. Enhanced optical nonlinearities using Rydberg EIT $^{15,22,23}$ have been observed in pioneering work ${ }^{13,14}$ that we are building upon. Very recently, the Rydberg blockade in a dense, mesoscopic atomic ensemble has been used to implement a deterministic single-photon source ${ }^{30}$. 
To observe the photon-photon blockade, several key requirements must be fulfilled. First, to eliminate Doppler broadening, the atoms should be cold so that they move by less than an optical wavelength during the microsecond lifetime of the EIT coherence. Second, the atomic cloud should be sufficiently dense such that the blockade condition $r_{b} \gtrsim l_{a}$ is fulfilled. Finally, the system should be one-dimensional, i.e. the transverse size of the probe beam should be smaller than the blockade radius $r_{b}$ in order to prevent polaritons from traveling side by side.

We fulfill these conditions by trapping a dense laser-cooled atomic ensemble and focusing the probe beam to a Gaussian waist $w=4.5 \mu \mathrm{m}<r_{b}$ (see Methods). We prepare an ${ }^{87} \mathrm{Rb}$ ensemble containing up to $10^{5}$ atoms in a far-detuned optical dipole trap produced by a Nd:YAG laser operating at $1064 \mathrm{~nm}$ with a total power of 5W. The trap is formed by two orthogonally polarized beams with waists $w_{t}=50 \mu \mathrm{m}$ intersecting at an angle of $32^{\circ}$. The atoms are optically pumped into the state $|g\rangle=\left|5 S_{1 / 2}, F=2, m_{F}=2\right\rangle$ in the presence of a $3.6 \mathrm{G}$ magnetic field along the quantization axis defined by the common propagation direction of the probe and control beams along the long axis of the cloud. The probe beam on the $|g\rangle \rightarrow|e\rangle=\left|5 P_{3 / 2}, F=3, m_{F}=3\right\rangle$ transition and the control beam on the $|e\rangle \rightarrow|r\rangle=\left|n S_{1 / 2}, J=\frac{1}{2}, m_{J}=\frac{1}{2}\right\rangle$ transition with waist $w_{c}=12.5 \mu \mathrm{m}$ are oppositely circularly polarized. The resonant optical depth of the cloud can be as large as $O D=50$, with in-trap radial and axial rms cloud dimensions of $\sigma_{r}=10 \mu \mathrm{m}$ and $\sigma_{a x}=36 \mu \mathrm{m}$, respectively. To avoid inhomogeneous light-shift broadening of the two-photon transition, we turn off the optical dipole trap before turning on the probe and control light, and probe the Rydberg EIT system continuously for up to a few hundred microseconds. The control light is filtered out from the transmitted light, and the photon-photon correlation function $g^{(2)}(\tau)$ of the 
probe beam is measured by means of two photon counters. The slow-light group delay $\tau_{d}$ through the atomic medium ${ }^{5}$ is measured independently in a pulsed experiment, and used to calculate the corresponding minimum group velocity $v_{g}=\sqrt{2 \pi} \sigma_{a x} / \tau_{d}$.

Probe transmission spectra are presented in Fig. 2a for large optical depth $O D=40$ and the control laser tuned to the Rydberg state $\left|100 S_{1 / 2}\right\rangle$. At very low incident photon rate $R_{i} \leq 1 \mu \mathrm{s}^{-1}$, the spectrum displays an EIT transparency window with $60 \%$ transmission. The transmission is mainly limited by the finite EIT decoherence rate $\gamma_{g r}$, which for our system is dominated by Doppler broadening and laser linewidth. The extraordinary nonlinearity of the Rydberg EIT medium ${ }^{13}$ becomes apparent as the incident photon rate is increased: the probe beam is strongly attenuated already at a photon flux of $R_{i} \sim 4 \mu \mathrm{s}^{-1}$. To demonstrate that we are operating in a quantum nonlinear regime, we show in Fig. 2b the correlation function $g^{(2)}(\tau)$ of the transmitted probe light, measured at $R_{i}=1.2 \mu \mathrm{s}^{-1}$. For the most strongly interacting state $\left|100 S_{1 / 2}\right\rangle$ with $r_{b}=13 \mu \mathrm{m} \approx 5 l_{a} \approx 2.9 w$ we observe strong antibunching with $g^{(2)}(0)=0.13(2)$, largely limited by background light. Subtraction of the independently measured background coincidence counts yields a corrected $g_{c}^{(2)}(0)=0.04(3)$. These observations are in stark contrast to EIT transmission via a less strongly interacting Rydberg state $\left|46 S_{1 / 2}\right\rangle$ with $r_{b}=3 \mu \mathrm{m}$, where the photon statistics of the transmitted light are similar to those of the incident coherent state (see inset). Interestingly, for $\left|100 S_{1 / 2}\right\rangle$ the photons are anti-bunched over a length scale $v_{g} \tau \sim 50 \mu \mathrm{m}$ that exceeds the blockade radius (see top axis of Fig. 2b), indicating the influence of additional propagation effects beyond the simple picture outlined above. 
To investigate the transmission characteristics of multiple photons through the medium, we plot in Fig. 3a the output photon rate $R_{o}$, scaled by the EIT transmission measured at low probe power, as a function of incident photon rate $R_{i}$. At first, $R_{o}$ increases linearly with $R_{i}$ as expected, but then saturates abruptly to a constant value of $R_{o}=1.3(3) \mu \mathrm{s}^{-1}$. Note that these observations deviate from the simplistic model of a multiphoton absorber that transmits only the one-photon component from the incoming coherent state (black dashed line). At the same time, the observed output flux corresponds to less than one photon in the medium $\left(R_{o}^{-1}>\tau_{d}=300 \mathrm{~ns}\right)$. Figure $3 \mathrm{~b}$ shows the saturated output rate versus the ratio $r_{b} / w$ of blockade radius and probe beam waist for a wide range of principal quantum numbers, control field intensities, and optical depths. The approximate $R_{o} \propto\left(w / r_{b}\right)^{2}$ scaling, valid for $w \gtrsim r_{b}$, indicates that the saturated rate for intermediate to strong interactions, $r_{b} \gtrsim l_{a}$, is largely determined by the transverse geometrical constraint, i.e. by the extent to which the Rydberg polaritons can propagate side by side through the medium.

Two important features of the photon-photon blockade are the degree of two-photon suppression at equal times, $g^{(2)}(0)$, and the associated correlation time, i.e., the width $\tau_{c}$ of the antibunching feature in $g^{(2)}(\tau)$. As discussed in detail below, the blockade mechanism is most effective if the optical depth per blockade radius $O D_{b}=r_{b} / l_{a}$ exceeds unity ${ }^{15}$, and if the system is effectively one-dimensional, $r_{b}>w$. Since the blockade radius ${ }^{27}$ increases with the principal quantum number $n$ as $r_{b} \propto n^{11 / 6}$, the combination of both effects results in a steep dependence of $g^{(2)}(0)$ upon $n$. Figures 4a,b show that $g^{(2)}(0)$ improves with principal quantum number $n$ of the Rydberg state and interaction strength $r_{b} / l_{a}$, resulting in a more than tenfold suppression of the two-photon transmission, limited by independently measured background light on the photon detectors (dotted lines). 
At the same time, the observed width $\tau_{c}$ of the $g^{(2)}$ feature considerably exceeds the photon travel time $\tau_{b}=r_{b} / v_{g} \sim 50 \mathrm{~ns}$ through the blockade radius (Figure $4 \mathrm{c}, \mathrm{d}$ ). Close examination (Fig. $4 \mathrm{~d}$ ) reveals that the correlation time is of the same order and scales inversely proportionally with the spectral width $^{5} B=\gamma_{\mathrm{EIT}} / \sqrt{8 O D}$ of the EIT transparency window. This observation suggests that propagation effects play an important role in establishing the $g^{(2)}$ correlation time $\tau_{c}$ in a medium of large optical depth. Remarkably, we observe that, under appropriate conditions, two photonevents are suppressed inside the medium on a length scale that approaches the size $\sigma_{a x} \sim 40 \mu \mathrm{m}$ of the entire atomic ensemble, and on a time scale that approaches the intrinsic coherence time $\gamma_{g r}^{-1}=500 \mathrm{~ns}$

To gain further understanding of these observations, we theoretically analyze the photon propagation dynamics in the weak-probe limit where the average number of photons inside the medium is much less than one. In this case, it suffices to consider two polaritons (Fig. 1b). The corresponding two-photon component of the state vector ${ }^{15}$ is $\left|\psi_{2}(t)\right\rangle=\frac{1}{2} \int d \mathbf{r}_{1} d \mathbf{r}_{2} E E\left(\mathbf{r}_{1}, \mathbf{r}_{2}, t\right) \hat{\mathcal{E}}^{\dagger}\left(\mathbf{r}_{1}\right) \hat{\mathcal{E}}^{\dagger}\left(\mathbf{r}_{2}\right)|0\rangle$, where $\hat{\mathcal{E}}(\mathbf{r})$ denotes the photon field operator, and $\left|E E\left(\mathbf{r}_{1}, \mathbf{r}_{2}, t\right)\right|^{2}$ is the probability of finding two photons at locations $\mathbf{r}_{1}, \mathbf{r}_{2}$. This probability directly yields the spatially dependent photon-photon correlation function, and, via the group velocity $v_{g}$, the corresponding temporal correlation function $g^{(2)}(\tau)$. An intuitive picture emerges if we make the simplification of a tightly focused probe beam (1D approximation) traveling through a homogeneous medium with perfect linear EIT transmission. In this case, the steady-state twophoton amplitude in the medium obeys (see SI):

$$
\partial_{R} E E\left(z_{1}, z_{2}\right)=-\frac{\mathcal{V}(r)}{l_{a}} E E\left(z_{1}, z_{2}\right)+4 l_{a}\left[1+\mathcal{V}(r) \frac{\Omega_{c}^{2}}{\Gamma^{2}}\right] \partial_{r}^{2} E E\left(z_{1}, z_{2}\right)
$$


where $R=\left(z_{1}+z_{2}\right) / 2$ and $r=z_{1}-z_{2}$ are the center-of-mass and relative coordinates of the two photons, respectively. The function $\mathcal{V}(r)=r_{b}^{6} /\left(r_{b}^{6}-2 i r^{6}\right)$ can be regarded as an effective potential that describes the impact of Rydberg-Rydberg interactions ${ }^{15}$. For large photon-photon distances, $r \gg r_{b}$, the potential $\mathcal{V}$ vanishes, and equation (1) yields perfect transmission under EIT, while for distances $r \lesssim r_{b}$, the interaction $\mathcal{V}$ modifies the two-photon propagation. According to equation (1), photon correlations emerge from a combination of two processes: the first term acts inside the blockade radius $r_{b}$ and describes absorption with a coefficient $l_{a}^{-1}$ as the interaction $\mathcal{V}$ tunes EIT out of resonance. This would create a sharp dip in the two-photon correlation function with a corresponding correlation time $\tau_{b}=r_{b} / v_{g}$ associated with the blockade radius. However, if the corresponding spectral width $\sim \tau_{b}^{-1}$ exceeds the spectral width $B$ of the EIT transparency window ${ }^{5}$, the second diffusion-like term acts to broaden the absorption dip (Fig. 1c) in space and time, increasing the photon-photon correlation time $\tau_{c}$ beyond $\tau_{b}$ towards a value set by the EIT transparency width (Fig. 4d). To maintain strong two-photon suppression $\left(g^{(2)}(0) \ll 1\right)$ in the presence of EIT-induced diffusion, the loss term must exceed the diffusion on the length scale of the blockade radius, requiring $r_{b}>l_{a}$. Large optical depth $O D_{b}=r_{b} / l_{a}$ of the blockaded region is therefore the key experimental feature that allows us to extend the earlier studies ${ }^{13,14}$ into the quantum nonlinear regime.

For direct comparisons with our experiments, we numerically solve the full set of propagation equations accounting for the Gaussian density profile of the trapped atomic cloud, the finite waist of the probe beam, and the imperfect single-photon transmission due to finite decoherence $\gamma_{g r}$ of the two-photon transition. As shown in Figures 2 and 4, the theory captures the essential 
features of our measured correlation functions and, moreover, reproduces their dependence on the Rydberg states, control laser intensities and optical depths of the sample over a wide range of parameters. This detailed theoretical understanding also allows us to analyze the prospects for possible future improvements. These include a reduction of Doppler broadening (through lower atomic temperature or the use of counterpropagating probe and control beams) to increase the linear transmission from $60 \%$ towards unity, the excitation of even higher-lying Rydberg states for larger blockade radius, and larger atomic density to further increase the optical depth per blockade radius $O D_{b}$ and overall optical depth $O D$.

Our observations open intriguing prospects for ultimate quantum control of light quanta. For example, by storing a single photon in a Rydberg state and subsequently transmitting a second Rydberg polariton, a single-photon switch can be created $^{15}$. It can be used, e.g., for quantum non-demolition measurements of optical photons. At the same time, by using strong interactions in the dispersive regime, the present approach can be used to implement deterministic quantum logic gates ${ }^{15,16}$, which would constitute a major advance towards all-optical quantum information processing $^{31}$. Finally, our results may open the door for exploring quantum dynamics of strongly interacting photonic many-body systems. For example, it may be possible to create a crystalline state of strongly interacting polaritons ${ }^{17}$. Beyond these specific applications, our work demonstrates that unique quantum nonlinear optical materials can be created by combining slow-light propagation with strong atom-atom interactions, an approach which can be potentially extended to realize other material systems with quantum nonlinearities. 


\section{Methods}

An ensemble of $6 \times 10^{6}$ laser-cooled atoms is captured in a magneto-optical trap (MOT) every $300 \mathrm{~ms}$. The trapped cloud is compressed and loaded into the dipole trap by the combined actions of increasing the magnetic-field gradient to $35 \mathrm{G} / \mathrm{cm}$, detuning the MOT trapping frequency by $-30 \mathrm{MHz}$ and reducing the MOT repumper intensity to $10 \mu \mathrm{W} / \mathrm{cm}^{2}$. The magnetic fields are then rapidly shut off, allowing for $10 \mathrm{~ms}$ of molasses cooling to a temperature of $35 \mu \mathrm{K}$. The crossed dipole trap holds up to $10^{5}$ atoms at a peak density of $2 \times 10^{12}$ atoms $/ \mathrm{cm}^{3}$ and a measured optical depth of $O D=50$.

The probe beam is focused to a $1 / e^{2}$ waist $w=4.5 \mu \mathrm{m}$ by a confocal arrangement of achromatic doublet lenses with focal length $30 \mathrm{~mm}$ and diameter $6.25 \mathrm{~mm}$. The control field is copropagating with the probe beam. The frequencies of both lasers are locked to an optical FabryPerot resonator that is stabilized against long-term drifts to a Doppler-free atomic resonance line. The measured short-term linewidths are $120 \mathrm{kHz}$ and $80 \mathrm{kHz}$ for the probe and control laser, respectively. The transmitted control light is separated from the probe light by a combination of interference and absorption filters.

The intensity correlation function of the outgoing probe field is measured with two singlephoton detectors. Spurious detection events typically limit $g_{2}(\tau) \geq 0.1$. These include dark counts from the detector, imperfect polarization of the probe photons (light with the orthogonal circular polarization is only weakly absorbed by the medium) and residual control light.

1. Yamamoto, Y. \& Imamoglu. Mesoscopic Quantum Optics (John Wiley \& Sons, Inc., New York, 1999).

2. Birnbaum, K. M. et al. Photon blockade in an optical cavity with one trapped atom. Nature 436, 87-90 (2005). 
3. Hau, L. V., Harris, S. E., Dutton, Z. \& Behroozi, C. H. Light speed reduction to 17 metres per second in an ultracold atomic gas. Nature (London) 397, 594-598 (1999).

4. Fleischhauer, M. \& Lukin, M. D. Dark-state polaritons in electromagnetically induced transparency. Phys. Rev. Lett. 84, 5094 - 5097 (2000).

5. Fleischhauer, M., Imamoglu, A. \& Marangos, J. P. Electromagnetically induced transparency: Optics in coherent media. Rev. Mod. Phys. 77, 633-673 (2005).

6. Tong, D. et al. Local blockade of rydberg excitation in an ultracold gas. Phys. Rev. Lett. 93, 063001 (2004).

7. Singer, K., Reetz-Lamour, M., Amthor, T., Marcassa, L. G. \& Weidemüller, M. Suppression of excitation and spectral broadening induced by interactions in a cold gas of rydberg atoms. Phys. Rev. Lett. 93, 163001 (2004).

8. Liebisch, T. C., Reinhard, A., Berman, P. R. \& Raithel, G. Atom counting statistics in ensembles of interacting rydberg atoms. Phys. Rev. Lett. 95, 253002 (2005).

9. Heidemann, R. et al. Rydberg excitation of bose-einstein condensates. Phys. Rev. Lett. 100, 033601 (2008).

10. Johnson, T. A. et al. Rabi oscillations between ground and rydberg states with dipole-dipole atomic interactions. Phys. Rev. Lett. 100, 113003 (2008).

11. Urban, E. et al. Observation of rydberg blockade between two atoms. Nat. Phys. 5, 110-114 (2009). 
12. Gaetan, A. et al. Observation of collective excitation of two individual atoms in the rydberg blockade regime. Nat. Phys. 5, 115-118 (2009).

13. Pritchard, J. D. et al. Cooperative atom-light interaction in a blockaded rydberg ensemble. Phys. Rev. Lett. 105, 193603 (2010).

14. Pritchard, J. D., Weatherill, K. J. \& Adams, C. S. Non-linear optics using cold rydberg atoms. arXiv:1205.4890v1 .

15. Gorshkov, A. V., Otterbach, J., Fleischhauer, M., Pohl, T. \& Lukin, M. D. Photon-photon interactions via rydberg blockade. Phys. Rev. Lett. 107, 133602 (2011).

16. Shahmoon, E., Kurizki, G., Fleischhauer, M. \& Petrosyan, D. Strongly interacting photons in hollow-core waveguides. Phys. Rev. A 83, 033806 (2011).

17. Chang, D. E., Gritsev, V., Morigi, G., Vuletic, M. D., V.and Lukin \& Demler, E. A. Crystallization of strongly interacting photons in a nonlinear optical fibre. Nat. Phys. 4, 884-889 (2008).

18. Schuster, I. et al. Nonlinear spectroscopy of photons bound to one atom. Nature Phys. 4, 382-385 (2008).

19. Fushman, I. et al. Controlled phase shifts with a single quantum dot. Science 320, 769-772 (2008).

20. Reinhard, A. et al. Strongly correlated photons on a chip. Nat. Photon. 6, 93-96 (2012). 
21. Tanji-Suzuki, H., Chen, W., Landig, R., Simon, J. \& Vuletić, V. Vacuum-induced transparency. Science 333, 1266-1269 (2011).

22. Petrosyan, D., Otterbach, J. \& Fleischhauer, M. Electromagnetically induced transparency with rydberg atoms. Phys. Rev. Lett. 107, 213601 (2011).

23. Sevinçli, S., Henkel, N., Ates, C. \& Pohl, T. Nonlocal nonlinear optics in cold rydberg gases. Phys. Rev. Lett. 107, 153001 (2011).

24. Saffman, M., Walker, T. G. \& Mølmer, K. Quantum information with rydberg atoms. Rev. Mod. Phys. 82, 2313-2363 (2010).

25. Møller, D., Madsen, L. B. \& Mølmer, K. Quantum gates and multiparticle entanglement by rydberg excitation blockade and adiabatic passage. Phys. Rev. Lett. 100, 170504 (2008).

26. Müller, M., Lesanovsky, I., Weimer, H., Büchler, H. P. \& Zoller, P. Mesoscopic rydberg gate based on electromagnetically induced transparency. Phys. Rev. Lett. 102, 170502 (2009).

27. Lukin, M. D. et al. Dipole blockade and quantum information processing in mesoscopic atomic ensembles. Phys. Rev. Lett. 87, 037901 (2001).

28. Bajcsy, M. et al. Efficient all-optical switching using slow light within a hollow fiber. Phys. Rev. Lett. 102, 203902 (2009).

29. Venkataraman, V., Saha, K., Londero, P. \& Gaeta, A. L. Few-photon all-optical modulation in a photonic band-gap fiber. Phys. Rev. Lett. 107, 193902 (2011). 
30. Dudin, Y. O. \& Kuzmich, A. Strongly interacting rydberg excitations of a cold atomic gas. Science 336, 887-889 (2012).

31. Han, Y., He, B., Heshami, K., Li, C.-Z. \& Simon, C. Quantum repeaters based on rydbergblockade-coupled atomic ensembles. Phys. Rev. A 81, 052311 (2010).

Acknowledgements We acknowledge technical support from Anton Mazurenko and partial support from NSF, CUA, and the AFOSR Quantum Memories MURI. AVG acknowledges funding from the Lee A. DuBridge Foundation and the IQIM, an NSF Physics Frontiers Center with support of the Gordon and Betty Moore Foundation.

Author Contributions The experiment was designed and built by S.H., T.Pe. and Q.L. Measurements and analysis of the data presented were carried out by T.Pe., O.F. and Q.L. The theoretical analysis was performed by A.V.G. and T.Po. All experimental and theoretical work was supervised by M.D.L. and V.V. All authors discussed the results and contributed to the manuscript.

Author Information Reprints and permissions information is available at www.nature.com/reprints. The authors declare that they have no competing financial interests. Correspondence and requests for materials should be addressed to M.D.L. (email: lukin@fas.harvard.edu) and V.V. (email: vuletic@mit.edu). 
Figure 1: Rydberg-blockade-mediated interaction between slow photons. a, b, An elongated ensemble of laser-cooled rubidium atoms is prepared in a crossed optical-dipole trap. Copropagating control and probe fields couple the ground-state $|g\rangle$ to a high-lying Rydberg state $|r\rangle$ via a short-lived excited state $|e\rangle$. Under EIT conditions, the probe photons slowly propagate in the medium as Rydberg polaritons. The Rydberg-Rydberg atom interaction $V(r)=\hbar C_{6} / r^{6}$ shifts the Rydberg levels out of resonance and blocks simultaneous Rydberg excitations at close range. Consequently two Rydberg polaritons cannot both propagate when they are closer than the blockade radius $r_{b}=\left(2 C_{6} / \gamma_{\mathrm{EIT}}\right)^{1 / 6}$, set by $V\left(r_{b}\right)=\hbar \gamma_{\mathrm{EIT}} / 2$, where $\gamma_{\mathrm{EIT}}=\Omega_{c}^{2} / \Gamma$ is the single-atom EIT linewidth as set by the control field Rabi frequency $\Omega_{c}$ and the decay rate $\Gamma$ of state $|e\rangle$. c, d, Numerical simulations showing the spatial evolution of the probability distribution associated with two photons (c) and two Rydberg excitations (d) at positions $\left(z_{1}, z_{2}\right)$ inside the medium, normalized by their values in the absence of blockade. Two Rydberg excitations are excluded from the blockaded range, resulting in the formation of an anti-bunching feature in the light field, whose width increases during the propagation due to the finite EIT transparency width $B=\gamma_{\mathrm{EIT}} / \sqrt{8 O D}$. 
Figure 2: Two-photon optical nonlinearity. a, Transmission spectra versus probe detuning at various incoming photon rates: $R_{i}=1 \mu \mathrm{s}^{-1}, 2 \mu \mathrm{s}^{-1}, 4 \mu \mathrm{s}^{-1}, 6 \mu \mathrm{s}^{-1}$ (dashed green, solid red, dotted blue, and dot-dashed black, respectively) for $\left|100 S_{1 / 2}\right\rangle$, EIT linewidth $\gamma_{\mathrm{EIT}}=2 \pi \times 23 \mathrm{MHz}$, optical depth $O D=40$, and measured group delay $\tau_{d}=250 \mathrm{~ns}$. The system is strongly nonlinear at a power as low as $0.25 \mathrm{pW}$. b, Photon-photon correlation function $g^{(2)}(\tau)$ at EIT resonance for the same parameters as in a with $R_{i}=1.2 \mu \mathrm{s}^{-1}$. The top axis shows the spatial separation $v_{g} \tau$ of polaritons with $v_{g} \approx 400 \mathrm{~m} / \mathrm{s}$. The error bars indicate $1 \sigma$ statistical uncertainty. Spurious detection events set a lower bound on $g^{(2)}$ of $0.09(3)$ (red dotted line). The inset shows $g^{(2)}(\tau)$ for the less strongly interacting state $\left|46 S_{1 / 2}\right\rangle$ with similar parameters. The solid lines are theoretical calculations as described in the text, with the probe waist fixed at $w=6 \mu \mathrm{m}$. Values $g^{(2)}>1$ are attributed to classical fluctuations (see Fig. S4 and SI). 
Figure 3: Saturation behavior of the transmission. a, Outgoing versus incoming photon rate for $\left|100 S_{1 / 2}\right\rangle, \gamma_{E I T}=2 \pi \times 15 \mathrm{MHz}, O D=26$, and a measured width $\tau_{c}=130 \mathrm{~ns}$ of the anti-bunching feature in $g^{(2)}(\tau)$. All output rates are scaled by the transmission of $50 \%$ at low photon rate due to linear absorption, and corrected for the finite detection-path efficiency. The dashed black curve outlines the expected rate if all multi-photon events in a time range $\tau=320 \mathrm{~ns}$ are fully blocked, while the green dashed curve assumes that all multiphoton states within $\tau=800 \mathrm{~ns}$ are converted into an outgoing one-photon state. $\mathbf{b}$, Saturated rate of outgoing photons $R_{o} \tau_{c}$ per anti-bunching correlation time $\tau_{c}$, scaled by the linear absorption, as a function of the ratio between the blockade radius $r_{b}$ and the probe beam waist $w$. The Rydberg states are $\left|100 S_{1 / 2}\right\rangle$ (blue, $w=4.5 \mu \mathrm{m}$, $O D_{b} \sim 8$; pink, $w=4.5 \mu \mathrm{m}, O D_{b} \sim 4$ ), $\left|77 S_{1 / 2}\right\rangle$ (black, $w=4.5 \mu \mathrm{m}, O D_{b} \sim 3$ ), $\left|46 S_{1 / 2}\right\rangle$ (green, $w=4.5 \mu \mathrm{m}, O D_{b} \sim 0.7$; red, $w=7 \mu \mathrm{m}, O D_{b} \sim 0.7$ ). $\tau_{c}$ is estimated using the single-atom EIT linewidths $\left(\gamma_{E I T}^{\square}, \gamma_{E I T}^{\diamond}, \gamma_{E I T}^{\circ}, \gamma_{E I T}^{\diamond}\right)=2 \pi \times(6-16,18-26,29-36,50) \mathrm{MHz}$, and varies from 60 to $330 \mathrm{~ns}$. The dashed line corresponds to $0.9\left(w / r_{b}\right)^{2}$, indicating the expected scaling with transverse confinement for $w \gtrsim r_{b}$. 
Figure 4: Dependence of the correlation function on EIT parameters. a, b, Equal-time photonphoton correlation $g^{(2)}(0)$ as a function of $O D$ for $\left|77 S_{1 / 2}\right\rangle$ (a) and $\left|100 S_{1 / 2}\right\rangle$ (b), for a set of single-atom EIT linewidths $\left(\gamma_{E I T}^{\bigcirc}, \gamma_{E I T}^{\nabla}, \gamma_{E I T}^{\triangleleft}, \gamma_{E I T}^{\triangleleft}\right)=2 \pi \times(20,27,16,26)$ MHz. Solid lines are numerical solutions for a probe beam waist $w=6 \mu \mathrm{m}$ including detection noise (dotted lines). c, d, Width $\tau_{c}$ of the anti-bunching feature in $g^{(2)}(\tau)$ as a function of optical depth (c) and EIT transparency width (d) $B=\gamma_{E I T} / \sqrt{8 O D}$, respectively. The black dashed line is $1.05 / B$ and derives from an approximate analytical solution of Equation (1) (see SI). The error bars indicate $1 \sigma$ statistical uncertainty. 